\title{
The mechanism of discrimination between oxidized and reduced coenzyme in the aldehyde dehydrogenase domain of Aldh111
}

\author{
Yaroslav Tsybovsky ${ }^{1}$, Yuryi Malakhau, Kyle C. Strickland, Sergey A. Krupenko* \\ Department of Biochemistry and Molecular Biology, Medical University of South Carolina, Charleston, SC 29425, USA
}

\section{A R T I C L E I N F O}

\section{Article history}

Available online 5 January 2013

\section{Keywords:}

Aldehyde dehydrogenase

10-Formyltetrahydrofolate dehydrogenase

$\mathrm{NADP}^{+} / \mathrm{NADPH}$

Catalytic residues

Coenzyme conformation

Crystal structure

\begin{abstract}
A B S T R A C T
Aldh111, also known as 10-formyltetrahydrofolate dehydrogenase (FDH), contains the carboxy-terminal domain $\left(\mathrm{C}_{\mathrm{t}}-\mathrm{FDH}\right)$, which is a structural and functional homolog of aldehyde dehydrogenases (ALDHs). This domain is capable of catalyzing the $\mathrm{NADP}^{+}$-dependent oxidation of short chain aldehydes to their corresponding acids, and similar to most ALDHs it has two conserved catalytic residues, Cys707 and Glu673. Previously, we demonstrated that in the $\mathrm{C}_{\mathrm{t}}$-FDH mechanism these residues define the conformation of the bound coenzyme and the affinity of its interaction with the protein. Specifically, the replacement of Cys707 with an alanine resulted in the enzyme lacking the ability to differentiate between the oxidized and reduced coenzyme. We suggested that this was due to the loss of a covalent bond between the cysteine and the $\mathrm{C} 4 \mathrm{~N}$ atom of nicotinamide ring of $\mathrm{NADP}^{+}$formed during $\mathrm{C}_{\mathrm{t}}$-FDH catalysis. To obtain further insight into the functional significance of the covalent bond between Cys707 and the coenzyme, and the overall role of the two catalytic residues in the coenzyme binding and positioning, we have now solved crystal structures of $\mathrm{C}_{\mathrm{t}}$-FDH in the complex with thio-NADP ${ }^{+}$and the complexes of the C707S mutant with NADP ${ }^{+}$and NADPH. This study has allowed us to trap the coenzyme in the contracted conformation, which provided a snapshot of the conformational processing of the coenzyme during the transition from oxidized to reduced form. Overall, the results of this study further support the previously proposed mechanism by which Cys707 helps to differentiate between the oxidized and reduced coenzyme during ALDH catalysis.
\end{abstract}

(c) 2013 Elsevier Ireland Ltd. All rights reserved.

\section{Introduction}

Aldehyde dehydrogenases are a family of $\operatorname{NAD}(\mathrm{P})^{+}$-utilizing enzymes that catalyze the conversion of a variety of aldehydes to their corresponding carboxylic acids. In humans, this family is represented by 19 distinct genes, which encode protein products that form homodimers or homotetramers [1]. ALDHs have a very similar structure, consisting of catalytic, nucleotide binding and oligomerization domains [2-8]. While structural boundaries between these domains can be clearly seen, the functional separation between them is somewhat arbitrary. For example, the nicotinamide ring of bound $\mathrm{NAD}(\mathrm{P})^{+}$protrudes into the catalytic domain to come in close proximity to catalytic residues. Of note, the nicotinamide moiety forms only a few contacts with an ALDH molecule

Abbreviations: ALDH, aldehyde dehydrogenase; Aldh111, 10-formyltetrahydrofolate dehydrogenase; $\mathrm{C}_{\mathrm{t}}-\mathrm{FDH}$, the carboxy-terminal ALDH domain of 10-formyltetrahydrofolate dehydrogenase.

* Corresponding author. Tel.: +1 843792 0845; fax: +1 8437928565

E-mail address: krupenko@musc.edu (S.A. Krupenko).

1 Present address: Department of Pharmacology, Case Western Reserve University, Cleveland, $\mathrm{OH}$ 44106, USA. and most of the interactions involve two ribose rings and the adenine moiety forming contacts with the protein within a classical Rossmann fold conserved throughout the family [3,5,6,8,9].

In addition to the structural conservation, there is also a functional conservation with regard to the ALDH mechanism. Thus, ALDHs have two highly conserved catalytic residues, a cysteine and a glutamate [1]. Numerous structural, site-directed mutagenesis and enzymatic studies of ALDHs have established the role of the cysteine as the active site nucleophile (reviewed in [1]). The proposed mechanism for ALDH catalysis includes two steps, (i) acylation and (ii) deacylation [3,5,10-13]. The cysteine forms a thiohemiacetal intermediate with the substrate during the acylation step, which requires deprotonation of its sulfhydryl group (reviewed in [1]). The glutamate has been proposed to facilitate the cysteine deprotonation, either directly or indirectly, and has also been suggested as a residue involved in the deacylation step of the reaction by activating a water molecule in the active site $[3,5,8,10,14,15]$. The dual role of the glutamate residue in combination with the conserved geometry of the active site created the requirement for coenzyme isomerization during catalysis [9]. In the first stage of the reaction the nicotinamide ring of the cofactor must be sufficiently close to the catalytic cysteine to receive the 
hydride. The corresponding stretched conformation of $\mathrm{NAD}(\mathrm{P})^{+}$ was named "extended". Once the hydride transfer is accomplished, the nicotinamide ring vacates the catalytic pocket to allow access of a water molecule that hydrolyzes the thioester intermediate, releasing the reaction product. This conformation of $\mathrm{NAD}(\mathrm{P}) \mathrm{H}$ was named "contracted" because the isomerization brings the nicotinamide moiety closer to the adenine part. In some crystal structures of ALDHs the nicotinamide ring of the reduced coenzyme is not seen due to disorder $[8,16]$. This conformation of $\mathrm{NADP}(\mathrm{H})$ is a functional analog of the contracted conformation, as it also allows space for the deacylation stage to proceed.

A member of the ALDH family, Aldh111 (FDH, 10-formyltetrahydrofolate dehydrogenase) is a folate-metabolizing enzyme, which is a product of a natural fusion of three unrelated genes [17]. One of these genes, an ancient aldehyde dehydrogenase, encodes for the carboxyl-terminal portion (residues 400-902, identified thereafter as $\mathrm{C}_{\mathrm{t}}-\mathrm{FDH}$ ) of Aldh111. This domain has high sequence similarity (up to 50\%) with ALDH1 and ALDH2, and can catalyze both $\mathrm{NADP}^{+}$-dependent $\mathrm{ALDH}$ or $\mathrm{NADP}^{+}$-independent esterase reactions using short-chain aldehydes as substrates [17-19]. Like many other ALDHs, $\mathrm{C}_{\mathrm{t}}$-FDH possesses two key catalytic residues, a cysteine (Cys707) and a glutamate (Glu673); replacement of either resulted in the catalytically inactive enzyme $[8,20]$. The ALDH reaction catalyzed by this domain is an integral part of the entire enzyme mechanism, which is the $\mathrm{NADP}^{+}$-dependent conversion of 10 -formyltetrahydrofolate to tetrahydrofolate and $\mathrm{CO}_{2}$ [17]. Of note, higher animals have a second gene encoding for a similar enzyme, Aldh1L2, which is a mitochondrial protein (in contrast to Aldh111, which is a cytosolic enzyme) [21,22].

Crystal structures of apo $\mathrm{C}_{\mathrm{t}}$-FDH and the holo enzyme in complexes with either $\mathrm{NADP}^{+}$or NADPH have demonstrated a high similarity of the protein molecule to other ALDHs [8]. $\mathrm{C}_{\mathrm{t}}$-FDH (as well as full-length Aldh111 [20]) forms a homotetramer, with each monomer displaying catalytic, coenzyme binding and oligomerization domains. Previous studies have demonstrated an unusual feature of the complex of $\mathrm{C}_{\mathrm{t}}$-FDH with the oxidized coenzyme: a transient covalent bond between the sulfur atom of the catalytic cysteine and the $\mathrm{C} 4 \mathrm{~N}$ atom of the nicotinamide (Fig. 1 ) [8,23]. Formation of such a bond has also been predicted for mitochondrial ALDH2 using in silico simulations [24] and was experimentally

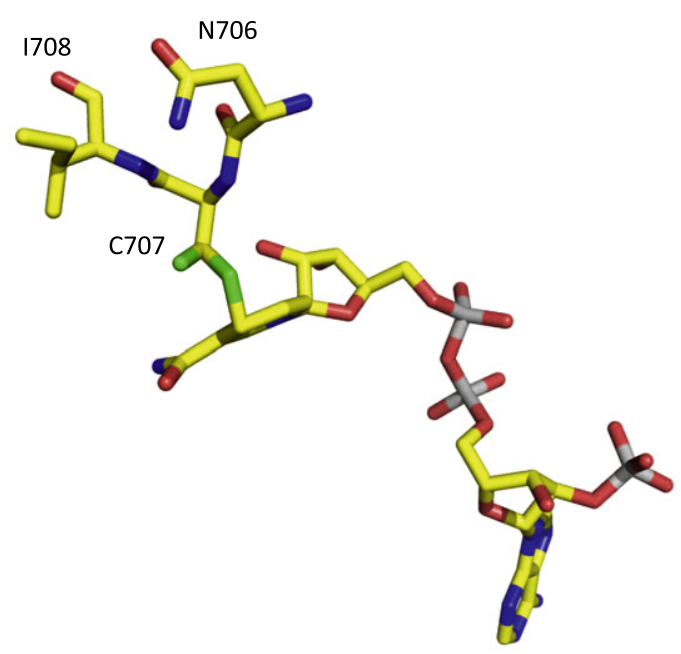

Fig. 1. The covalent bond between Cys707 of $C_{t}-F D H$ and $C 4 N$ of the nicotinamide ring of NADP ${ }^{+}$. Two positions of the sulfur, one forming the covalent bond (1.6 distance to $(4 \mathrm{~N}$ ) and another oriented away from the nicotinamide ring ( $4 \AA$ distance to $\mathrm{C} 4 \mathrm{~N}$ ) are shown (green). (For interpretation of the references to color in this figure legend, the reader is referred to the web version of this article.) shown for other ALDHs [25]. It has been further demonstrated that the ability to form such a covalent bond is important for the discrimination between oxidized and reduced coenzyme bound to $\mathrm{C}_{\mathrm{t}}$-FDH [23]. In particular, replacement of the catalytic cysteine of $\mathrm{C}_{\mathrm{t}}$-FDH with an alanine resulted in an enzyme that bound both $\mathrm{NADP}^{+}$and NADPH in the extended conformation. This study also suggested that the conserved catalytic glutamate controls the binding and discharging of the coenzyme, presumably through long-range communications with helix $G$ that interacts with its adenine moiety. In the present work we aimed to clarify the role of the cysteine and the functional significance of its covalent bond with $\mathrm{NADP}^{+}$in proper positioning of the coenzyme. We further wanted to elucidate if the nicotinamide ring of the coenzyme, which is known to only loosely bind to ALDHs, plays a significant part in defining the overall conformation of $\operatorname{NADP}(\mathrm{H})$. To this end, we solved crystal structures of the C707S mutant of $\mathrm{C}_{\mathrm{t}}-\mathrm{FDH}$ in complex with $\mathrm{NADP}^{+}$or NADPH and a structure of wild-type $\mathrm{C}_{\mathrm{t}}-\mathrm{FDH}$ in complex with thio-NADP ${ }^{+}$, an $\mathrm{NADP}^{+}$analog with an altered nicotinamide group. Here we report the structural analysis of these proteins with regard to coenzyme binding and effects of Cys707 and Glu673 on the conformation of bound dinucleotide, and we expand the model for the conformational processing of the coenzyme during the transition from oxidized to reduced form.

\section{Materials and methods}

\subsection{Protein preparation}

Site-directed mutagenesis was carried out using a QuickChange site-directed mutagenesis kit (Agilent Technologies) and confirmed by DNA sequencing of the mutant constructs. Wild type and mutant $\mathrm{C}_{\mathrm{t}}-\mathrm{FDH}$ were expressed in $E$. coli as constructs with $5 \times$ His tag at the amino-terminus and purified on Ni-NTA resin (GE-Healthcare) as we previously described [8].

\subsection{Crystallization and data collection}

Crystals were grown by the vapor diffusion method in hanging drops over wells containing $1.5-1.6 \mathrm{M}$ ammonium sulfate and $0.1 \mathrm{M}$ MES-NaOH, pH 6.4 or $0.1 \mathrm{M}$ HEPES-NaOH, pH 7.2, as described [8]. Crystals of binary complexes with NADP ${ }^{+}$, NADPH or thio-NADP were produced by overnight soaking of protein crystals in the presence of $5 \mathrm{mM}$ of the corresponding coenzyme. Prior to mounting, crystals were passed through the mother liquor solution supplemented with the corresponding coenzyme and containing $27.5 \%$ glycerol and flash-cooled in situ at $100 \mathrm{~K}$ using an X-Stream Cryostream (Rigaku MSC).

Data sets were collected on an RAXIS IV++ image plate detector mounted on a RU-H3R rotating anode X-ray generator operating at $50 \mathrm{kV}$ and $100 \mathrm{~mA}$ (Medical University of South Carolina). All the crystals belonged to space group $\mathrm{C} 2$ with cell dimensions $a=258.6, b=194.8, c=97.4$, and $\beta=108.8$. The data were processed using HKL2000 [26]. The statistics are shown in Table 1.

\subsection{Model building and refinement}

Structures were obtained by molecular replacement using Molrep [27] with the structure of apo $C_{t}-F D H$ (PDB code 2O2P) [8] as the search model. Coenzyme molecules were identified by examining the $\left|F_{O}\right|-\left|F_{C}\right|$ and $2\left|F_{O}\right|-\left|F_{C}\right|$ electron density maps. Models were refined by alternating rounds of manual building using $\mathrm{O}$ [28] and Coot [29] and restrained refinement in REFMAC5 [30]. Water molecules were introduced with Coot [29] and verified manually. During refinement, $5 \%$ of the data were reserved to calculate the free R-factor. Table 1 shows the refinement statistics. 
Table 1

Data collection and refinement statistics.

\begin{tabular}{llll}
\hline & C707S NADP $^{+}$ & C707S NADPH & WT Thio-NADP \\
\hline Resolution $(\AA)$ & 2.3 & 3.4 & 2.3 \\
Completeness \% & $95.9(94.3)$ & $98.3(97.9)$ & $100.0(100.0)$ \\
Mean $I / \sigma I$ & $7.7(1.7)$ & $6.4(2.1)$ & $11.7(1.9)$ \\
Redundancy & $2.0(2.0)$ & $2.5(2.4)$ & $2.9(2.8)$ \\
$\mathrm{R}_{\text {merge }}(\%)$ & $9.6(39.9)$ & $19.9(55.3)$ & $9.6(56.4)$ \\
$\mathrm{R}_{\text {cryst }}(\%)$ & 18.1 & 19.8 & 17.7 \\
$\mathrm{R}_{\text {free }}(\%)$ & 21.0 & 25.2 & 19.6 \\
Rmsd bond lengths $(\AA)$ & 0.012 & 0.018 & 0.012 \\
Rmsd bond angles $\left({ }^{\circ}\right)$ & 1.37 & 1.92 & 1.38 \\
Ramachandran plot: & & & \\
\% Favored & 97.1 & 91.0 & $97.7 \%$ \\
\% Allowed & 2.7 & 7.9 & $2.1 \%$ \\
$\%$ Outliers & 0.2 & 1.1 & $0.2 \%$ \\
\hline
\end{tabular}

The figures in parentheses refer to the highest resolution shell of the data.

Stereochemistry of the models was verified by PROCHECK [31]. The coordinates and structure factors have been deposited in the Protein Data Bank with codes 4GNZ, 4GO0 and 4GO2 for the C707S in complex with $\mathrm{NADP}^{+}$, the C707S in complex with NADPH and wild type $\mathrm{C}_{\mathrm{t}}-\mathrm{FDH}$ in complex with thio-NADP ${ }^{+}$, respectively. Figures were prepared using PyMol (www.pymol.org).

\subsection{Assay of ALDH activity}

The ALDH activity of $\mathrm{C}_{\mathrm{t}}$-FDH and the C707S mutant was measured by following the increase in absorbance at $340 \mathrm{~nm}$ as a result of formation of NADPH. The standard assay contained $22 \mu \mathrm{g} / \mathrm{ml}$ the enzyme, $120 \mu \mathrm{M} \mathrm{NADP}{ }^{+}$, and $5 \mathrm{mM}$ propanal in $50 \mathrm{mM}$ HEPES$\mathrm{NaOH}$ buffer, $\mathrm{pH}$ 9.0. The reaction was initiated by the addition of propanal. All measurements were done at $25{ }^{\circ} \mathrm{C}$ using a Shimadzu 2401PC double beam spectrophotometer. The activity of wild type $\mathrm{C}_{\mathrm{t}}$-FDH with thio-NADP ${ }^{+}$(Sigma) was measured by monitoring the increase in absorbance at $398 \mathrm{~nm}$ as a result of formation of thio$\mathrm{NADPH}$.

\subsection{Coenzyme binding study}

Wild type $\mathrm{C}_{\mathrm{t}}-\mathrm{FDH}$ or the mutated proteins were incubated with increasing concentrations of $\mathrm{NADP}^{+}$(up to $4.2 \times 10^{-5} \mathrm{M}$ ), NADPH (up to $5 \times 10^{-5} \mathrm{M}$ ) or thio-NADP (up to $4.2 \times 10^{-5} \mathrm{M}$ ) for $1 \mathrm{~h}$ at room temperature in $50 \mathrm{mM}$ Tris- $\mathrm{HCl}$ buffer, $\mathrm{pH} 7.5$ containing $100 \mathrm{mM} \mathrm{NaCl}$ and $0.5 \mathrm{mM}$ DTT. Emission fluorescence spectra of proteins were recorded on a Hitachi F-2500 fluorescence spectrophotometer by scanning from 300 to $400 \mathrm{~nm}$ with excitation at $295 \mathrm{~nm}$. Changes in NADPH fluorescence at $450 \mathrm{~nm}$ were recorded upon excitation at $330 \mathrm{~nm}$. $K_{\mathrm{d}}$ values were determined by non-linear fitting of experimental data to equations described in our previous publication [23].

\section{Results}

\subsection{Overall structure}

All crystals obtained in this study belong to the $\mathrm{C} 2$ space group with unit cell dimensions identical to those of the wild type $\mathrm{C}_{\mathrm{t}}-\mathrm{FDH}$ crystals [8]. Protein subunits share the same fold and show no appreciable structural rearrangements compared to these previously published models.

\subsection{Structures of the C707S mutant of $C_{t}-F D H$ in complex with $N A D P^{+}$ and $N A D P H$}

We determined two structures of the C707S mutant of $\mathrm{C}_{\mathrm{t}}-\mathrm{FDH}$ the complex with $\mathrm{NADP}^{+}$at $2.3 \AA$ and the complex with NADPH at $3.4 \AA$ resolution. These structures show no significant conformational deviations from wild type $\mathrm{C}_{\mathrm{t}}-\mathrm{FDH}$, and their active sites are virtually identical (Fig. 2). Similarly to the structure of the wildtype enzyme with bound NADP ${ }^{+}[8]$ and to the structure of the corresponding mutant of human mitochondrial ALDH [9], in the C707S-NADP ${ }^{+}$complex the coenzyme is bound in the extended conformation, with the nicotinamide ring displacing the side chain of the catalytic Glu673 to attain close proximity to Ser707 (Fig. 2A). However, the serine residue in place of the catalytic cysteine does not form a covalent bond with the $\mathrm{C} 4 \mathrm{~N}$ atom of the nicotinamide ring.

A remarkable feature of the C707S-NADPH complex is the extended conformation of the reduced coenzyme (Fig. 2B). In contrast, in the wild type $\mathrm{C}_{\mathrm{t}}$-FDH the nicotinamide ring of the reduced coenzyme is disordered, presumably due to the lack of contacts with the protein [8]. We have previously demonstrated
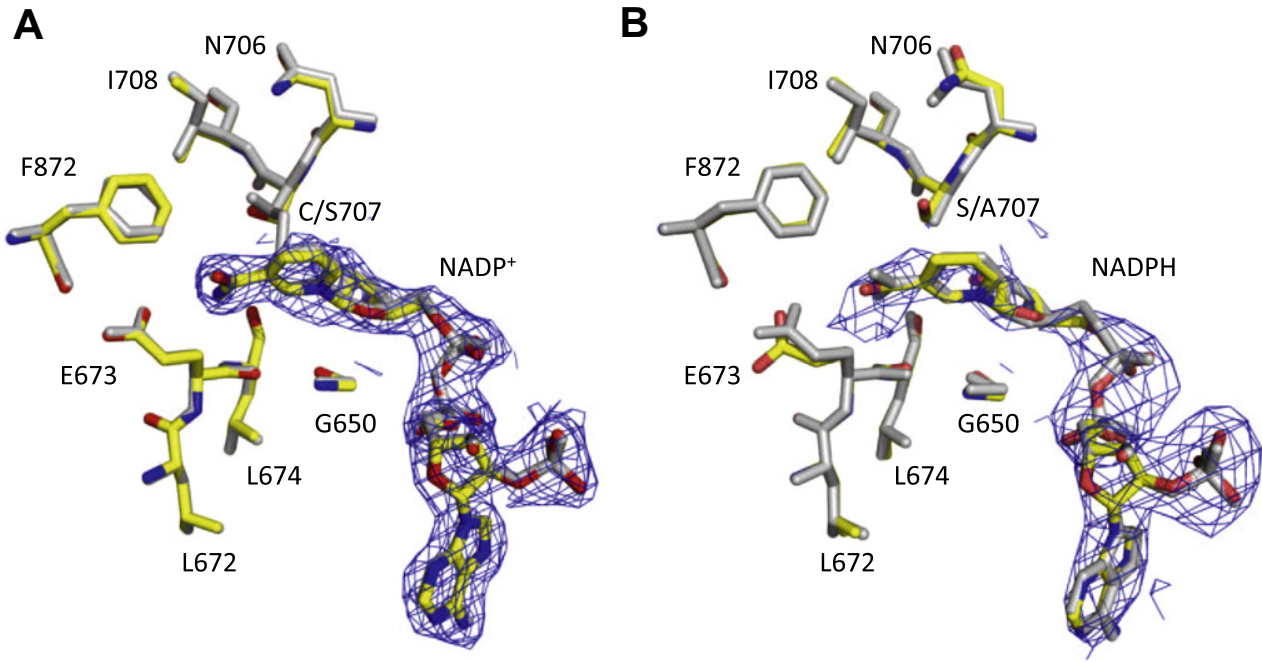

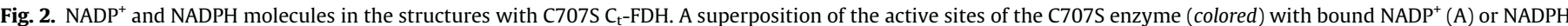

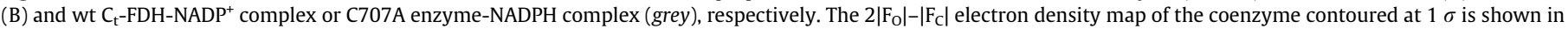
blue. (For interpretation of the references to color in this figure legend, the reader is referred to the web version of this article.) 
the identical arrangement of the reduced coenzyme (extended conformation) bound to another $\mathrm{C}_{\mathrm{t}}-\mathrm{FDH}$, the C707A enzyme [23]. In that enzyme, however, the extended conformation of the reduced coenzyme could be possible because the smaller side-chain of alanine allows additional space in the catalytic pocket for the nicotinamide ring. Here our data demonstrated that despite a higher structural and chemical similarity between a serine and a cysteine versus an alanine and a cysteine, NADPH still is in the extended conformation when bound to the C707S enzyme. Of note, the average distance between the hydroxyl oxygen of Ser707 and the C4N atom of NADPH in this structure is $2.9 \pm 0.2 \AA$, which provides enough space to accommodate the nicotinamide ring. Thus, the sulfur atom of Cys707 (likely due to the ability to form the transient covalent bond with the $\mathrm{C} 4 \mathrm{~N}$ atom of nicotinamide) seems to be crucial for discriminating between the oxidative states of the coenzyme. This sensory function either allows the oxidized nicotinamide ring to enter the catalytic pocket or repels the reduced ring.

\subsection{Structure of wild type $C_{t}-F D H$ in complex with thio-NADP $P^{+}$}

We used a coenzyme analog, thio-NADP ${ }^{+}$(Fig. 3), to further probe the role of the two catalytic residues, Cys707 and Glu673, in the coenzyme binding. The structure of wild-type $\mathrm{C}_{\mathrm{t}}-\mathrm{FDH}$ in complex with thio-NADP ${ }^{+}$, determined at $2.3 \AA$ resolution, maintains the same protein fold, but the coenzyme is bound in two different conformations with approximately equal occupancies. While one of these conformers is mostly disordered, the other forms a "crooked" structure with the nicotinamide part showing minimal contacts with the protein (Fig. 4A). In this new conformation the nicotinamide ring is shifted $4.6 \AA$ outward from the catalytic center, and it closely resembles the canonical contracted conformation of NADH in the complex with ALDH2 [9] (Fig. 4B). Furthermore, as was previously shown in the structure of the $\mathrm{C}_{\mathrm{t}^{-}}$<smiles></smiles>

$\mathrm{NADP}^{+}$<smiles></smiles>

Thio-NADP ${ }^{+}$
Fig. 3. Comparison of structures of $\mathrm{NADP}^{+}$(left) and thio-NADP${ }^{+}$(right).
$\mathrm{FDH} / \mathrm{NADP}^{+}$complex, binding of the nicotinamide ring in the extended conformation of the coenzyme is associated with a movement of the side chain of Glu673 out of the active site (Fig. 4C) [8]. In this new position, Glu673 forms tight contacts with several other residues, water molecules and the carboxamide group of the coenzyme (Fig. 4C). In contrast, in the structure of the $\mathrm{C}_{\mathrm{t}}$-FDH/thioNADP $^{+}$complex the side chain of Glu673 remains in contact with Cys707 as was shown previously for the ligand-free structure [8], blocking the entrance to the catalytic center (Fig. 4A). It is likely that the bulky sulfur atom of thio-NADP ${ }^{+}$substituting for the carboxamide oxygen of $\mathrm{NADP}^{+}$cannot be accommodated in the extended conformation, and its repulsion from the carboxyl group of the glutamate results in the nicotinamide moiety being expelled from the active site. Of note, thio-NADP ${ }^{+}$appears in the extended conformation in the structures of DHFR [32] and transhydrogenase [33], the fact suggesting that this conformation is possible. Thus, we suggest that thio-NADP ${ }^{+}$bound to $\mathrm{C}_{\mathrm{t}}-\mathrm{FDH}$ represents the conformation of the natural coenzyme on its way out after the completion of the hydride transfer.

\subsection{Enzymatic activity and coenzyme binding}

We have previously demonstrated that C707A $\mathrm{C}_{\mathrm{t}}-\mathrm{FDH}$ is catalytically inactive [20]. A similar effect was observed when the catalytic cysteine of other aldehyde dehydrogenases was replaced with alanine [34]. However, replacement of the cysteine with a serine in mitochondrial rat liver aldehyde dehydrogenases (Aldh2) resulted in an enzyme that retained residual catalytic activity [34]. In the present study, analysis of the C707S $\mathrm{C}_{\mathrm{t}}$-FDH revealed that it was catalytically inactive. We have also determined $K_{\mathrm{d}} \mathrm{s}$ for binding both oxidized and reduced coenzymes by the mutant enzyme. The $K_{\mathrm{d}}$ for $\mathrm{NADP}^{+}$was determined based on quenching of tryptophan fluorescence of the C707S enzyme upon binding of the coenzyme as we previously described [20]. The decrease in fluorescence was likely due to quenching of fluorescence of two tryptophans near the coenzyme binding site, $\operatorname{Trp} 573$ located about $4 \AA$ from the pyrophosphate group of the coenzyme and Trp582 located about $8 \AA$ from the nicotinamide ring. The replacement of Cys707 with a serine resulted in a significant decrease in the affinity for coenzyme with $K_{\mathrm{d}}$ of $11.0 \pm 1.5 \mu \mathrm{M}$ ( $K_{\mathrm{d}}$ for the wild-type enzyme is $0.9 \pm 0.1 \mu \mathrm{M}$ ). The above method cannot be used to measure the $K_{\mathrm{d}}$ for NADPH binding due to the fluorescence resonance energy transfer from the tryptophan residues to NADPH [23]. Therefore, changes in NADPH fluorescence at $450 \mathrm{~nm}$ were
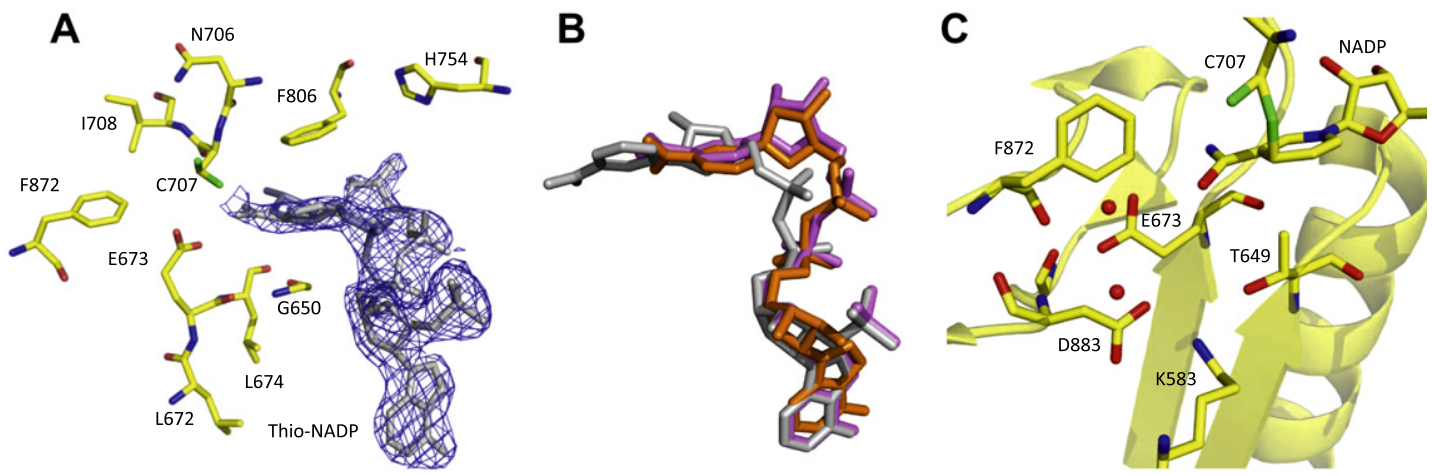

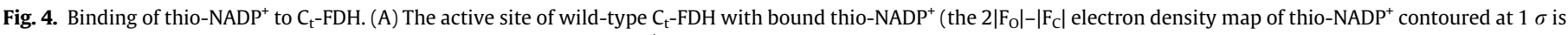

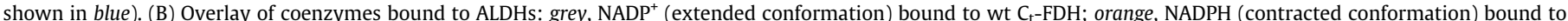

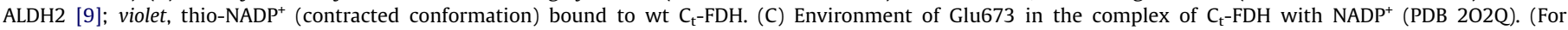
interpretation of the references to color in this figure legend, the reader is referred to the web version of this article.) 
monitored after excitation at $330 \mathrm{~nm}$ (binding to $\mathrm{C}_{\mathrm{t}}$-FDH leads to enhanced NADPH fluorescence) [35]. These experiments demonstrated that the wild-type and C707S enzymes have similar $K_{\mathrm{d}}$ values for NADPH (data not shown). This is in contrast to C707A enzyme, which binds NADPH more tightly than the wild-type enzyme does [23]. We suggest the difference is due to the larger side chain of the serine as compared to the alanine residue.

We have also evaluated the aldehyde dehydrogenase activity of wild-type $\mathrm{C}_{\mathrm{t}}-\mathrm{FDH}$ using thio-NADP ${ }^{+}$as the coenzyme. The enzyme did not exhibit any catalytic activity with this derivative, a finding that is in agreement with our structural data demonstrating that the nicotinamide moiety is positioned too far from the catalytic cysteine to allow the hydride transfer; the closest distance between the $\mathrm{C} 4 \mathrm{~N}$ atom of the nicotinamide of thio-NADP ${ }^{+}$and the sulfur of the catalytic cysteine is $4.5 \AA$ compared to $1.6 \AA$ in the case of $\mathrm{NADP}^{+}$(Fig. 1) [8]. The $K_{\mathrm{d}}$ value $(17.0 \pm 0.8 \mu \mathrm{M})$ for the binding of thio-NADP ${ }^{+}$evaluated by quenching of tryptophan fluorescence was significantly increased compared to the $K_{\mathrm{d}}$ value for wild-type enzyme.

\section{Discussion}

Nicotinamide dinucleotide molecules, $\mathrm{NAD}^{+} / \mathrm{NADH}$ and $\mathrm{NADP}^{+} /$ $\mathrm{NADPH}$, transfer a hydride ion in oxido-reductive reactions and are among the most common coenzymes found in the cell, being utilized by several hundreds of different enzymes [36,37]. While the precise mode of the accommodation of these coenzymes in their respective binding pockets varies among enzymes and is defined by structural arrangements of catalytic and nucleotide binding centers, the enzyme mechanism, and by specific residues involved in the coenzyme binding and catalysis [38], there are several common features of dinucleotide binding. For example, the most common structural motif involved in the binding of $\mathrm{NAD}(\mathrm{P})^{+}$molecules is the Rossmann fold (reviewed in [36,39]), which is found in many different classes of dehydrogenases including the ALDH family [2,3]. Furthermore, while the nicotinamide adenine dinucleotide is a flexible molecule that can adopt different conformations depending on its environment [40], the coenzyme is bound to most proteins preferentially in the extended conformation [38]. An interesting feature of the coenzyme binding by ALDHs, though, is two distinct conformations of the dinucleotide molecule, extended and contracted [2,3, $5,8,9,41]$. It is generally accepted that the former represents the hydride transfer position of the oxidized coenzyme in the acylation step of the catalysis while the latter corresponds to the reduced coenzyme in the second, deacylation, step when the nicotinamide ring has already left the catalytic pocket.

Crystal structures of the holo $\mathrm{C}_{\mathrm{t}}-\mathrm{FDH}$ in complex with $\mathrm{NADP}^{+}$or $\mathrm{NADPH}$ demonstrated two conformations of the dinucleotide
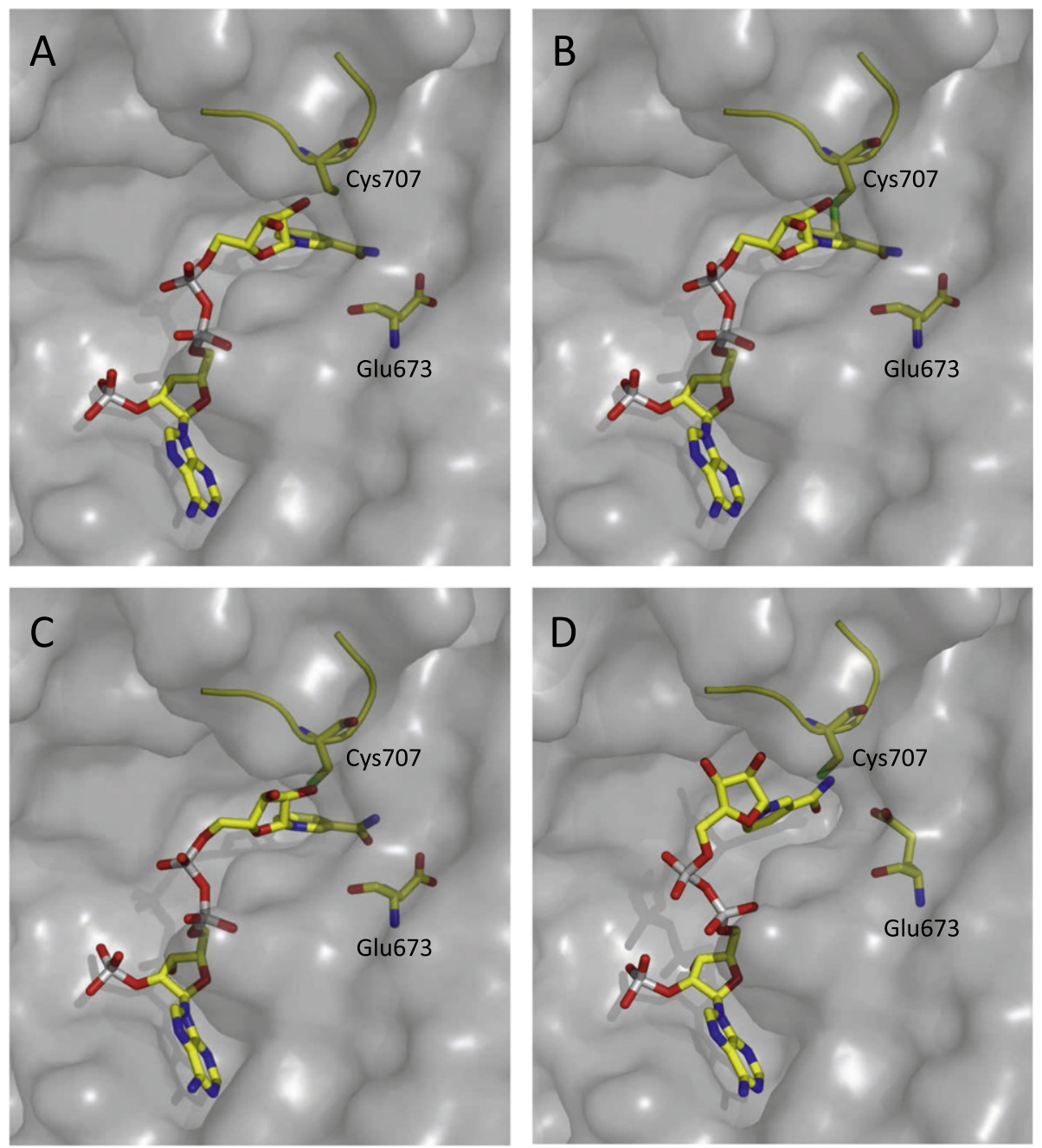

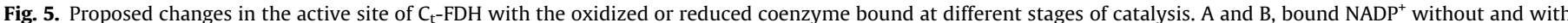

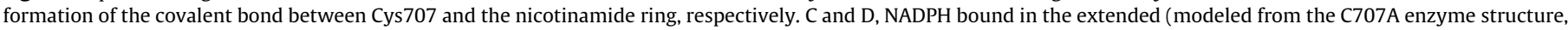
PDB 3RHR [23]) or contracted (modeled from wt $\mathrm{C}_{\mathrm{t}}-\mathrm{FDH} /$ thio-NADP ${ }^{+}$complex) conformation, correspondingly. 
molecule as well, an extended one for the oxidized coenzyme and one which was interpreted as the contracted conformation for the reduced coenzyme [8]. Of note, in that presumably contracted conformation, there was no electron density seen for the nicotinamide ring of NADPH, suggesting the flexibility of positioning of the reduced ring in this structure. Apparently, the nicotinamide ring in the oxidized coenzyme forms additional contacts with the enzyme that fix it in the extended conformation, whereas the lack of such contacts for the ring in the reduced coenzyme allows it to move into various positions. In the present study, however, the contracted conformation of the coenzyme, with a well-ordered nicotinamide ring, has been observed in the complex of $\mathrm{C}_{\mathrm{t}}-\mathrm{FDH}$ with thio-NADP ${ }^{+}$

Our recent studies of $\mathrm{C}_{\mathrm{t}}$-FDH shed light on the mechanism by which ALDHs could retain the oxidized coenzyme in the extended conformation and also discriminate against the binding of the reduced coenzyme [23]. This novel mechanism of the interaction between ALDHs and $\mathrm{NAD}(\mathrm{P})^{+}$involves a transient covalent bond formed between the catalytic nucleophile (the sulfhydryl group of the cysteine residue) and the $\mathrm{C} 4 \mathrm{~N}$ atom of the nicotinamide ring [8]. Since this finding was reported, covalent bonds between the catalytic cysteine and the nicotinamide ring have been observed in crystal structures of several other aldehyde dehydrogenases $[24,25]$, the fact implicating this phenomenon as an integral part of the catalytic mechanism. We initially suggested that such a covalent bond facilitates stronger binding of the coenzyme and the proper orientation of the nicotinamide ring within the catalytic center [8]. The follow up study from our laboratory has further demonstrated that the covalent bond between the cysteine and nicotinamide ring plays an additional role as the sensor recognizing the oxidative state of the coenzyme [23]. This hypothesis has received further support in the present study.

Based on our data, we propose the following mechanism for discharging the coenzyme from its binding site (schematically depicted in Fig. 5). This mechanism presents two catalytic residues, Cys707 and Glu673, as the key determinants defining the mode of interaction between the enzyme and the coenzyme, and can be described as a two-phase process: (i) the displacement of the reduced nicotinamide ring from the catalytic center, and (ii) dissociation of the coenzyme from the enzyme. In the first phase, upon binding the oxidized coenzyme, the sulfhydryl group of the cysteine forms a transient covalent bond with the $\mathrm{C} 4 \mathrm{~N}$ atom. This bond, which was also demonstrated for the hydrolytic ALDH from Pseudomonas aeruginosa, can easily dissociate via an elimination reaction [25]. The fact that we observed two positions of the sulfur atom of the cysteine with $50 \%$ occupancy might suggest that the formation and dissociation of this bond is a constant process. We hypothesize that the ability to form such a temporary bond serves as a sensor of the oxidized state of the coenzyme (it cannot be formed with the reduced form, NADPH). This step is schematically depicted in Fig. 5 (panels A and B) as the balance between two stages, with and without the covalent bond. In the second step of this phase, following the hydride transfer and the reduction of the coenzyme, the sulfhydryl group comes into steric hindrance with the nicotinamide ring (Fig. 5, panel C). In the third step, this hindrance results in the displacement of the ring from the catalytic center thus changing the coenzyme conformation from extended to contracted (Fig. 5, panel D).

In our crystal structures, the latter stage is characterized by the lack of well-defined electron density for the reduced nicotinamide ring, but well-defined density for the adenosine diphophosphoribose. We interpreted this conformation of NADPH as a disordered nicotinamide ring and the rest of the coenzyme rigidly bound to helix G, which itself is well-ordered at this stage (Fig. 6, panel A). Based on our previous studies [23], we hypothesize that such an arrangement (an ordered helix $G$ ) is caused by the distant effect of the catalytic glutamate, which at this point is displaced from the catalytic pocket by the nicotinamide ring (Fig. 6, panel A). The second phase of the coenzyme dissociation involves the rotation of the glutamate back into the nicotinamide-binding pocket within the catalytic center, which may initiate remote conformational alterations in helix $\mathrm{G}$ (as shown previously for the E673Q enzyme [23]) thus causing its disorder (Fig. 6, panel B). The disordered helix is losing contact with the adenine moiety, which
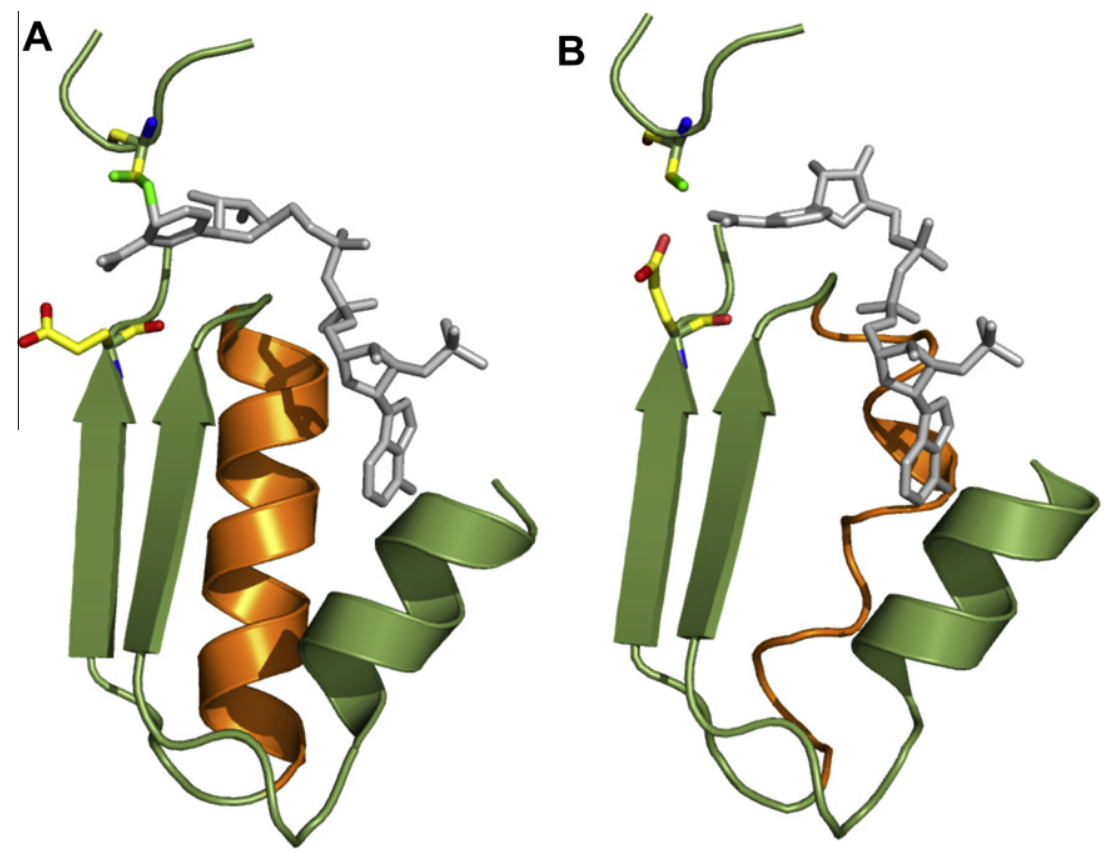

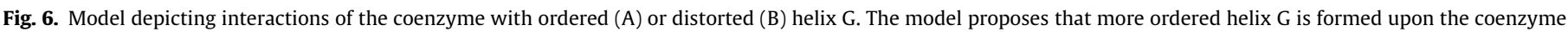

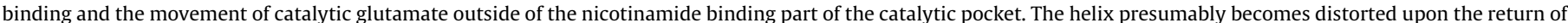

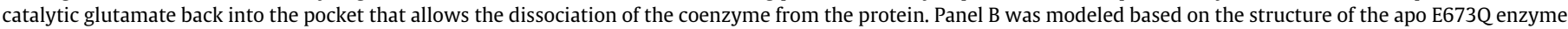
(PDB 3RHM [23]) and the structure of wt $\mathrm{C}_{\mathrm{t}}-\mathrm{FDH}$ in complex with thio-NADP ${ }^{+}$. 
allows coenzyme dissociation from the enzyme (there would be no strong contacts between the enzyme and the coenzyme with such an arrangement). It should be noted that Glu673 is also likely to participate in the first stage of coenzyme discharging, through energetically unfavorable interactions with carboxamide of nicotinamide, thus cooperating with Cys707 in dissociation of the nicotinamide from the catalytic center. In this regard, the glutamate could discriminate between different orientations of the planes of the carboxamide group relative to the nicotinamide ring in the oxidized and reduced coenzyme, a mechanism described for other enzymes [32,33].

It is not clear at present whether the proposed mechanism of discrimination between the reduce and oxidize states of the coenzyme is unique for $\mathrm{C}_{\mathrm{t}}-\mathrm{FDH}$, limited to certain members of ALDH family, or whether it is a more common phenomenon for this class of enzymes. In this regard, while the catalytic cysteine is conserved in all active aldehyde dehydrogenases, the glutamate is less conserved [42]. It is also not clear whether the mechanism is extended to other classes of oxido-reductases. Of note, the ALDH reaction is essentially unidirectional [43], whereas for example alcohol dehydrogenases catalyze a reversible reaction [44]. Thus, a specific feature of ALDH catalysis could be the necessity to discharge the reduced coenzyme without the requirement to bind and utilize it for the reverse reaction. Toward this end, our study underscores the role of the cysteine and glutamate in discriminating between the reduced and oxidized coenzyme, a feature that perhaps would not be required for enzymes catalyzing the reaction in both directions. Curiously, it appears that one of the ALDHs, ALDH18A1, catalyzes the reversal of ALDH reaction. ALDH18A1, an NADPHdependent $\gamma$-glutamyl phosphate reductase, is a part of the bifunctional pyrroline-5-carboxylate synthase, the key enzyme in the biosynthesis of proline and ornithine [45-48]. It is attractive to suggest that the reductase does not have the catalytic glutamate analogous to Glu673 of $\mathrm{C}_{\mathrm{t}}$-FDH and binds NADPH in the extended conformation. However, while the crystal structure of the apo enzyme from Thermotoga maritima has been solved [49], the structure of its complex with the coenzyme awaits future studies.

\section{Conflict of interest statement}

None.

\section{Acknowledgments}

The authors would like to thank Dr. John Hempel, University of Pittsburg for helpful discussions. This work was supported by The National Institutes of Health grant DK54388. Kyle C. Strickland was supported by a Ruth L. Kirschstein National Research Service Award for Individual Predoctoral MD/PhD Fellows F30DK083215. The X-ray crystallography facility used for this work is supported by the Medical University of South Carolina's Research Resource Facilities program.

\section{References}

[1] V. Koppaka, D.C. Thompson, Y. Chen, M. Ellermann, K.C. Nicolaou, R.O. Juvonen, D. Petersen, R.A. Deitrich, T.D. Hurley, V. Vasiliou, Aldehyde dehydrogenase inhibitors: a comprehensive review of the pharmacology, mechanism of action, substrate specificity, and clinical application, Pharmacol. Rev. 64 (2012) 520-539.

2] ZJ. Liu, YJ. Sun, J. Rose, Y.J. Chung C.D. Hsiao, W.R. Chang I. Kuo, J. Perozich, R. Lindahl, J. Hempel, B.C. Wang, The first structure of an aldehyde dehydrogenase reveals novel interactions between NAD and the Rossmann fold, Nat. Struct. Biol. 4 (1997) 317-326.

[3] C.G. Steinmetz, P. Xie, H. Weiner, T.D. Hurley, Structure of mitochondrial aldehyde dehydrogenase: the genetic component of ethanol aversion, Structure 5 (1997) 701-711.
[4] A.L. Lamb, M.E. Newcomer, The structure of retinal dehydrogenase type II at 2.7 A resolution: implications for retinal specificity, Biochemistry 38 (1999) 6003-6011.

[5] S.A. Moore, H.M. Baker, T.J. Blythe, K.E. Kitson, T.M. Kitson, E.N. Baker, Sheep liver cytosolic aldehyde dehydrogenase: the structure reveals the basis for the retinal specificity of class 1 aldehyde dehydrogenases, Structure 6 (1998) $1541-1551$.

[6] K. Johansson, M. El-Ahmad, S. Ramaswamy, L. Hjelmqvist, H. Jornvall, H. Eklund, Structure of betaine aldehyde dehydrogenase at 2.1 A resolution, Protein Sci. 7 (1998) 2106-2117.

[7] D. Cobessi, F. Tête-Favier, S. Marchal, S. Azza, G. Branlant, A. Aubry, Apo and holo crystal structures of an NADP-dependent aldehyde dehydrogenase from Streptococcus mutans, J. Mol. Biol. 290 (1999) 161-173.

[8] Y. Tsybovsky, H. Donato, N.I. Krupenko, C. Davies, S.A. Krupenko, Crystal structures of the carboxyl terminal domain of rat 10 -formyltetrahydrofolate dehydrogenase: implications for the catalytic mechanism of aldehyde dehydrogenases, Biochemistry 46 (2007) 2917-2929.

[9] S.J. Perez-Miller, T.D. Hurley, Coenzyme isomerization is integral to catalysis in aldehyde dehydrogenase, Biochemistry 42 (2003) 7100-7109.

[10] R.A. Muñoz-Clares, L. González-Segura, A.G. Díaz-Sánchez, Crystallographic evidence for active-site dynamics in the hydrolytic aldehyde dehydrogenases. Implications for the deacylation step of the catalyzed reaction, Chem. Biol. Interact. 191 (2011) 137-146.

[11] R.I. Feldman, H. Weiner, Horse liver aldehyde dehydrogenase. II. Kinetics and mechanistic implications of the dehydrogenase and esterase activity, J. Biol. Chem. 247 (1972) 267-272.

[12] S. Sheikh, L. Ni, T.D. Hurley, H. Weiner, The potential roles of the conserved amino acids in human liver mitochondrial aldehyde dehydrogenase, J. Biol. Chem. 272 (1997) 18817-18822.

[13] S. Marchal, D. Cobessi, S. Rahuel-Clermont, F. Tête-Favier, A. Aubry, G Branlant, Chemical mechanism and substrate binding sites of NADPdependent aldehyde dehydrogenase from Streptococcus mutans, Chem. Biol. Interact. 130-132 (2001) 15-28.

[14] X. Wang, H. Weiner, Involvement of glutamate 268 in the active site of human liver mitochondrial (class 2) aldehyde dehydrogenase as probed by sitedirected mutagenesis, Biochemistry 34 (1995) 237-243.

[15] S. Marchal, S. Rahuel-Clermont, G. Branlant, Role of glutamate-268 in the catalytic mechanism of nonphosphorylating glyceraldehyde-3-phosphate dehydrogenase from Streptococcus mutans, Biochemistry 39 (2000) 33273335.

[16] D. Cobessi, F. Tête-Favier, S. Marchal, G. Branlant, A. Aubry, Structural and biochemical investigations of the catalytic mechanism of an NADP-dependent aldehyde dehydrogenase from Streptococcus mutans, J. Mol. Biol. 300 (2000) 141-152.

[17] S.A. Krupenko, FDH: an aldehyde dehydrogenase fusion enzyme in folate metabolism, Chem. Biol. Interact. 178 (2009) 84-93.

[18] S.A. Krupenko, C. Wagner, R.J. Cook, Expression, purification, and properties of the aldehyde dehydrogenase homologous carboxyl-terminal domain of rat 10 formyltetrahydrofolate dehydrogenase, J. Biol. Chem. 272 (1997) 1026610272.

[19] K.C. Strickland, N.I. Krupenko, M.E. Dubard, C.J. Hu, Y. Tsybovsky, S.A. Krupenko, Enzymatic properties of ALDH1L2, a mitochondrial 10formyltetrahydrofolate dehydrogenase, Chem. Biol. Interact. 191 (2011) 129-136.

[20] S.A. Krupenko, C. Wagner, R.J. Cook, Cysteine 707 is involved in the dehydrogenase activity site of rat 10-formyltetrahydrofolate dehydrogenase, J. Biol. Chem. 270 (1995) 519-522.

[21] N.I. Krupenko, M.E. Dubard, K.C. Strickland, K.M. Moxley, N.V. Oleinik, S.A Krupenko, ALDH1L2 is the mitochondrial homolog of 10formyltetrahydrofolate dehydrogenase, J. Biol. Chem. 285 (2010) 2305623063.

[22] K.C. Strickland, R.S. Holmes, N.V. Oleinik, N.I. Krupenko, S.A. Krupenko, Phylogeny and evolution of aldehyde dehydrogenase-homologous folate enzymes, Chem. Biol. Interact. 191 (2011) 122-128.

[23] Y. Tsybovsky, S.A. Krupenko, Conserved catalytic residues of the ALDH1L1 aldehyde dehydrogenase domain control binding and discharging of the coenzyme, J. Biol. Chem. 286 (2011) 23357-23367.

24] T. Wymore, D.W. Deerfield 2nd, J. Hempel, Mechanistic implications of the cysteine-nicotinamide adduct in aldehyde dehydrogenase based on quantum mechanical/molecular mechanical simulations, Biochemistry 46 (2007) 94959506.

[25] A.G. Díaz-Sánchez, L. González-Segura, E. Rudiño-Piñera, A. Lira-Rocha, A Torres-Larios, R.A. Muñoz-Clares, Novel NADPH-cysteine covalent adduct found in the active site of an aldehyde dehydrogenase, Biochem. J. 439 (2011) 443-452.

[26] Z. Otwinowski, W. Minor, Processing of X-ray diffraction data collected in oscillation mode, Methods Enzymol. 276 (1997) 307-326.

[27] A. Vagin, A. Teplyakov, MOLREP: an automated program for molecular replacement, J. Appl. Crystallogr. 30 (1997) 1022-1025.

[28] T.A. Jones, J.Y. Zou, S.W. Cowan, M. Kjeldgaard, Improved methods for building protein models in electron density maps and the location of errors in these models, Acta Crystallogr. A 47 (Pt 2) (1991) 110-119.

[29] P. Emsley, K. Cowtan, Coot: model-building tools for molecular graphics, Acta Crystallogr. D Biol. Crystallogr. 60 (2004) 2126-2132.

[30] A.A. Vagin, R.A. Steiner, A.A. Lebedev, L. Potterton, S. McNicholas, F. Long, G.N. Murshudov, REFMAC5 dictionary: organization of prior chemical knowledge 
and guidelines for its use, Acta Crystallogr. D Biol. Crystallogr. 60 (2004) 2184 2195.

[31] R.A. Laskowski, M.W. MacArthur, D.S. Moss, J.M. Thornton, PROCHECK: a program to check the stereochemical quality of protein structures, J. Appl Crystallogr. 26 (1993) 283-291.

[32] M.A. McTigue, J.F. Davies 2nd, B.T. Kaufman, J. Kraut, Crystal structures of chicken liver dihydrofolate reductase: binary thioNADP ${ }^{+}$and ternary thioNADP ${ }^{+}$biopterin complexes, Biochemistry 32 (1993) 6855-6862.

[33] A. Singh, J.D. Venning, P.G. Quirk, G.I. van Boxel, D.J. Rodrigues, S.A. White, J.B. Jackson, Interactions between transhydrogenase and thio-nicotinamide analogues of $\mathrm{NAD}(\mathrm{H})$ and $\mathrm{NADP}(\mathrm{H})$ underline the importance of nucleotide conformational changes in coupling to proton translocation, J. Biol. Chem. 278 (2003) 33208-33216.

[34] J. Farrés, T.T. Wang, S.J. Cunningham, H. Weiner, Investigation of the active site cysteine residue of rat liver mitochondrial aldehyde dehydrogenase by sitedirected mutagenesis, Biochemistry 34 (1995) 2592-2598.

[35] K. Takahashi, H. Weiner, J.H. Hu, Increase in the stoichiometry of the functioning active sites of horse liver aldehyde dehydrogenase in the presence of magnesium ions, Arch. Biochem. Biophys. 205 (1980) 571-578

[36] C.R. Bellamacina, The nicotinamide dinucleotide binding motif: a comparison of nucleotide binding proteins, FASEB J. 10 (1996) 1257-1269.

[37] F. Mu, P.J. Unkefer, C.J. Unkefer, W.S. Hlavacek, Prediction of oxidoreductasecatalyzed reactions based on atomic properties of metabolites, Bioinformatics 22 (2006) 3082-3088

[38] G. Kuppuraj, K. Sargsyan, Y.H. Hua, A.R. Merrill, C. Lim, Linking distinct conformations of nicotinamide adenine dinucleotide with protein fold/ function, J. Phys. Chem. B 115 (2011) 7932-7939.

[39] H. Jörnvall, J. Hedlund, T. Bergman, U. Oppermann, B. Persson, Superfamilies SDR and MDR: from early ancestry to present forms. Emergence of three lines, a Zn-metalloenzyme, and distinct variabilities, Biochem. Biophys. Res. Commun. 396 (2010) 125-130.

[40] P.E. Smith, J.J. Tanner, Conformations of nicotinamide adenine dinucleotide $(\mathrm{NAD}(+))$ in various environments, J. Mol. Recognit. 13 (2000) 27-34.

[41] L. González-Segura, E. Rudiño-Piñera, R.A. Muñoz-Clares, E. Horjales, The crystal structure of a ternary complex of betaine aldehyde dehydrogenase from Pseudomonas aeruginosa provides new insight into the reaction mechanism and shows a novel binding mode of the $2^{\prime}$-phosphate of NADP and a novel cation binding site, J. Mol. Biol. 385 (2009) 542-557.

[42] J. Hempel, I. Kuo, J. Perozich, B.C. Wang, R. Lindahl, H. Nicholas, Aldehyde dehydrogenase. Maintaining critical active site geometry at motif 8 in the class 3 enzyme, Eur. J. Biochem. 268 (2001) 722-726.

[43] J. Hempel, S. Stanley, J. Perozich, T. Wymore, H.B. Nicholas Jr, Residue conservations in aldehyde dehydrogenase gene products reemphasize functional interpretations, in: E.M.H. Weiner, R. Lindahl, B. Plapp (Eds.), Enzymology and Molecular Biology of Carbonyl Metabolism, Purdue University Press, West Lafayette IN, 2006.

[44] G. Duester, J. Farres, M.R. Felder, R.S. Holmes, J.O. Hoog, X. Pares, B.V. Plapp, S.J. Yin, $H$. Jornvall, Recommended nomenclature for the vertebrate alcohol dehydrogenase gene family, Biochem. Pharmacol. 58 (1999) 389-395.

[45] I. Pérez-Arellano, F. Carmona-Alvarez, A.I. Martínez, J. Rodríguez-Díaz, J. Cervera, Pyrroline-5-carboxylate synthase and proline biosynthesis: from osmotolerance to rare metabolic disease, Protein Sci. 19 (2010) 372-382.

[46] C.A. Hu, S. Khalil, S. Zhaorigetu, Z. Liu, M. Tyler, G. Wan, D. Valle, Human delta1-pyrroline-5-carboxylate synthase: function and regulation, Amino Acids 35 (2008) 665-672.

[47] L.S. Bicknell, J. Pitt, S. Aftimos, R. Ramadas, M.A. Maw, S.P. Robertson, A missense mutation in ALDH18A1, encoding delta1-pyrroline-5-carboxylate synthase (P5CS), causes an autosomal recessive neurocutaneous syndrome, Eur. J. Hum. Genet. 16 (2008) 1176-1186.

[48] D.L. Skidmore, D. Chitayat, T. Morgan, A. Hinek, B. Fischer, A. Dimopoulou, G. Somers, W. Halliday, S. Blaser, Y. Diambomba, E.G. Lemire, U. Kornak, S.P. Robertson, Further expansion of the phenotypic spectrum associated with mutations in ALDH18A1, encoding delta(1)-pyrroline-5-carboxylate synthase (P5CS), Am. J. Med. Genet. A 155A (2011) 1848-1856.

[49] R. Page, M.S. Nelson, F. von Delft, M.A. Elsliger, J.M. Canaves, L.S. Brinen, X. Dai, A.M. Deacon, R. Floyd, A. Godzik, C. Grittini, S.K. Grzechnik, L. Jaroszewski, H.E. Klock, E. Koesema, J.S. Kovarik, A. Kreusch, P. Kuhn, S.A. Lesley, D. McMullan, T.M. McPhillips, M.D. Miller, A. Morse, K. Moy, J. Ouyang, A. Robb, K. Rodrigues, R. Schwarzenbacher, G. Spraggon, R.C. Stevens, H. van den Bedem, J. Velasquez, J. Vincent, X. Wang, B. West, G. Wolf, K.O. Hodgson, J. Wooley, I.A. Wilson, Crystal structure of gamma-glutamyl phosphate reductase (TM0293) from Thermotoga maritima at 2.0 A resolution, Proteins 54 (2004) 157-161. 